\section{PCa: Ibandronat gut bei Knochenschmerzen}

\author{
Bei lokalisierten Knochenschmerzen aufgrund von Metastasen eines \\ Prostatakarzinoms ist die Radiotherapie Standard. Nun wurde die Effektivität \\ einer einzelnen Infusion des Bisphosphonats Ibandronat getestet.
}

nochenmetastasen sind eine häufige

Komplikation beim Prostatakarzinom (PCa). Dass sich metastasenbedingte Knochenschmerzen mit Bisphosphonaten gut kontrollieren lassen, zeigten frühere Studien, ein direkter Vergleich zur Radiotherapie fehlte jedoch.

In einer Studie erhielten nun 470 Patienten mit metastasenbedingten Knochenschmerzen randomisiert entweder eine Einzeldosis Radiotherapie (8 Gy) oder eine einzelne Infusion von $6 \mathrm{mg}$ Ibandronat. Die Schmerzen wurden zu Beginn der Studie sowie nach 4, 8, 12, 26 und 52 Wochen mithilfe des BPI („brief pain inventory") erhoben. Wer innerhalb von vier Wochen nicht ansprach, durfte die jeweils andere Therapie durchführen. Anhand der Kriterien der World Health Organization (WHO) (pain ladder) und des EAS („effective analgesic score“) wurde die Schmerzreduktion über den Konsum von Analgetika und einen Schmerzscore im Vergleich zur Baseline ermittelt. Maximal erlaubt war eine Differenz von $\pm 15 \%$ zwischen den Gruppen. Komplettes Ansprechen war als Schmerzscore von null mit stabilem oder reduziertem Analgetikaverbrauch definiert. Als partielles Ansprechen galt eine Reduktion im Schmerzscore von mindestens zwei Punkten mit stabilem oder reduziertem Analgetikaverbrauch oder - bei einer Schmerzreduktion von höchstens einem Punkt - eine Reduktion des Analgetikaverbrauchs von mindestens $25 \%$.

Nach vier und zwölf Wochen gab es zwischen den Gruppen keine statistisch signifikanten Unterschiede in der Schmerzreduktion. Bei der Radiotherapie trat das Ansprechen schneller ein. Hinsichtlich der Stärke von Nebenwirkungen gab es keine Unterschiede, aber in den Therapiearmen traten unterschiedliche Nebenwirkungen auf. Die Lebensqualität war nach vier und zwölf Wochen vergleichbar. Auch das Gesamtüberleben war in beiden Gruppen ähnlich. Es war bei $\mathrm{Pa}$ tienten, die wegen fehlenden Ansprechens nach vier Wochen eine erneute Behandlung mit der jeweils anderen Therapie erhielten, besser als bei jenen, die auf eine weitere Behandlung verzichteten.

Fazit: Eine Einzelinfusion Ibandronat kann Knochenschmerzen infolge von Metastasen bei Prostatakarzinom ähnlich gut lindern wie eine einzelne Bestrahlung. Nach Ansicht der Forscher steht somit das Bisphosphonat als Option zur Verfügung, wenn eine Radiotherapie nicht möglich ist. Kathrin von Kieseritzky

Hoskin $\mathrm{P}$ et al. A multicenter randomized trial of Ibandronate compared with single-dose radiotherapy for localized metastatic bone pain in prostate cancer. J Natl Cancer Inst. 2015; 107(10): djv197.

\title{
Auch kleine Steinreste machen Ärger
}

\author{
Verbleiben nach endoskopischer Steinentfernung Reste in Harnleiter \\ oder Niere, besteht die Chance, dass diese auf natürlichem Weg \\ abgehen. Je nach Größe können sie aber auch eine Menge \\ Probleme bereiten, wie eine kanadische Studie nahelegt.
}

$\mathrm{R}_{4}^{\mathrm{s}}$ eststeinfragmente, die kleiner als $4 \mathrm{~mm}$ sind, gehen bei $26 \%$ der Patienten nach einer Ureteroskopie spontan ab. Wenn nicht, bleiben sie häufig symptomlos. Jedem fünften Patienten machen diese Überbleibsel jedoch zu schaffen. Ben Chew von der University of British Columbia, Vancouver, und Kollegen haben bei 232 Patienten mit Reststeinen den Verlauf im Anschluss an eine Ureteroskopie untersucht. Grundlage hierfür waren die Daten aus sechs Zentren der Endourology Disease Group for Excellence (EDGE) der Jahre 2006 bis 2013. Im Mittel wurden die Teilnehmer über 17 Monate nachbeobachtet.

56\% der Patienten blieben symptomfrei und benötigten keine weiteren Behandlungen. Bei $44 \%$ traten jedoch er- neut Steinbeschwerden auf. $15 \%$ aller Studienteilnehmer entwickelten Komplikationen, die keiner Intervention bedurften, bei $29 \%$ war eine Nachbehandlung erforderlich. Die Technik der primären Steinentfernung spielte dabei keine Rolle.

Obwohl rund $26 \%$ aller Steine nach der Ureteroskopie unabhängig von ihrer Größe spontan abgingen, waren die Komplikationsrate sowie die Notwendigkeit für einen erneuten Eingriff signifikant größenabhängig. Überschritten die Reststeinfragmente eine Größe von $4 \mathrm{~mm}$, nahmen sie im Verlauf häufiger weiter an Größe zu, die Komplikationsrate erreichte $59 \%$ (vs. $28 \%$ bei kleineren Steinresten) und die Reinterventionsrate $38 \%$ (vs. $18 \%$ ).

Eine Subanalyse zeigte: Fragmente über $2 \mathrm{~mm}$ wuchsen zwar auch, führten aber nicht zu Komplikationen und erforderten keine erneuten Eingriffe.

Fazit: Chew und seine Mitarbeiter schließen aus ihren Ergebnissen, dass eine Restfragmentgröße von mehr als $4 \mathrm{~mm}$ nach Ureteroskopie ein erhöhtes Risiko für erneute Steinbildung, mehr Komplikationen (17,2 \% Notfallbehandlungen, 15,1 \% erneute Koliken, 6,5\% Klinikeinweisungen, 2,6\% Niereninsuffizienz) und eine höhere Reinterventionsrate nach sich zieht. Patienten mit Reststeingrößen über $4 \mathrm{~mm}$ sollten, den Urologen zufolge, deshalb präventiv behandelt werden. Aber auch Fragmente unter $4 \mathrm{~mm}$ sollten nachbeobachtet werden.

Die effektivste Strategie, um mögliche Probleme nach einer Ureteroskopie zu vermeiden, sei jedoch die Steine komplett zu entfernen. Dr. Christine Starostzik

Chew B H et al. Natural history, complications, and re-intervention rates of asymptomatic residual stone fragments post-ureteroscopy: a report from the EDGE Research Consortium. J Urol. 2015;doi:10.1016/j.juro.2015.11.009. 